\title{
Exploration to the Issues Associated with Different Coverage Methods in WSN
}

\author{
Amit Sharma, \\ Department of Computer Science and Technology, \\ ITMU, Gurgaon, India
}

\begin{abstract}
A Sensor network is defined as the critical situational network in which, some critical nodes are defined with energy constraints. To improve the network reliability, such kinds of networks are defined under different coverage methods. These methods includes area coverage, target coverage etc. The area coverage is effective to reduce the chances of orphan nodes under limited sensing range analysis. In same way, target coverage is effective to provide backup to critical nodes. In this work, various issues associated with various coverage methods are explored.
\end{abstract}

\section{Keywords}

Issues, Orphan Nodes, Target Coverage, Area Coverage

\section{INTRODUCTION}

Coverage is having its significance to improve the communication reliability in constraint specific sensor network. The network is defined with the restricted specification in terms of energy constraints, sensing range etc.

Coverage is a fundamental issue in a WSN, which determines how well a phenomenon of interest (area or target) is monitored or tracked by sensors [4]. Effectiveness of coverage system in a sensor network will improve the QoS for different network operations so that the improvement to network localization and clustering will be achieved. The core phenomenon defined with coverage is to identify the existence of some node in the sensing range so that effective communication and monitoring will be performed. It is also effective to improve the network reliability because of high connectivity ratio. Additionally the coverage formulations try and realize the weak points i.e. the points that area unit least lined by the devices in a very sensor field and recommend future readying and reconfiguration schemes for rising the coverage performance. Usually the coverage involves the two basic ideas [5]:

- The analysis of coverage performance once the device nodes square measure deployed within the observation region.

- The improvement of the coverage performance once the device network cannot effectively satisfy the applying needs.

Sensing coverage characterizes the monitoring quality provided by a sensor network in a designated region. Different applications require different degree of sensing coverage. There are various associated application depends on coverage range analysis so that network monitoring and node tracking will be done effectively. The effectiveness of this node tracking depends on associated parameters including the energy specification, fading rate and failure probability. These parameters collectively increase the network strength and tolerance under different deficiencies. The requirement vectors for the network are also changed because of these parameters. The coverage requirement may also change after a network has been deployed, for instance, due to changes in application modes or environmental conditions. Each sensor node is able to sense the phenomenon in a definite sensing area. Any point in the sensing area of a sensor is said to be secured by the sensor the sensing area of a sensor is normally assumed to be a circle with the sensor located at the core. The radius of the disk is called the sensing range of the sensor. Considering the coverage concept, the formulation of network can be improved so that network life will be improved and the energy consumption over the network will be reduced [1][5][6].

Coverage issues square measure basic and crucial in planning a wireless device network. The coverage drawback of the WSN square measure to hide the whole space or set of explicit targets among wherever we would wish to scrutiny in associate degree continuous amount by deploying copious sensors haphazardly. It's caused by three main reasons: not enough sensors to hide the full Region of Interest (ROI), restricted sensing vary and random preparation. As sensors square measure operated exploitation restricted power provide a number of them die out leading to inadequate sensors to totally cowl the ROI.A device's sensing vary is confined to an explicit radius thanks to that sensor cannot cowl the region outside its sensing vary which ends up to the coverage drawback.. All aspects of network coverage includes area coverage, target coverage and barrier coverage are affected through various associated factors including the energy, failure rate and connectivity vectors. Depended on the coverage objectives and applications, they can be roughly classified [6] on the basis of what is to be covered, namely area coverage, Target coverage and Barrier coverage.

- Target Coverage Problems: there exists an arrangement of predetermined targets which need to be checked (secured) in an altered conveyed area. Because of limited battery vitality of sent sensors, target coverage issues are focusing on designing successful scheduling algorithms to draw out the time for monitoring these targets.

- Area Coverage Problems: In an interested area we have to guarantee that each point of the entire area can be checked by at least one sensor. The coverage issue is to maximize the time for monitoring the entire area.

- Barrier Coverage Problems: Given a barrier we want to guarantee that each article moves across the barrier will be recognized by the sent sensors.

Among these issues, area coverage issues have already received extensive attention in past years. On the other hand other two problems begin to draw attention recently. Our work focuses on target coverage problem in a sensor network. Coverage is in general associated with energy-efficiency and 
network connectivity, two important properties of WSN.Various algorithms has been proposed to solve the above coverage problems. Our research focus on the following considerations: evaluating and improving coverage performance for target coverage, while maximizing the network lifetime.

\section{LITERATURE SURVEY}

Lot of work is already done, in the area of area coverage by different researchers in the form of localization algorithms. Some of the work defined by earlier researchers is defined here under

Author [1] discussed the types of coverage problem according to different standards. They contemplated the sorts of the coverage issue according the organization of the networks, the characters of observed areas or targets, the sensing models of sensor hubs and so on. To enhance the coverage performance an algorithm is also proposed in this paper. Using the versatility of hubs, the algorithm can move redundant hubs to revealed area although there are a few points of confinement of vitality and hub hardware, the algorithm is still powerful in practice. Author[2] review the common strategies used in solving coverage problem in WSN. They reviewed the researches done in maximizing coverage of WSN by sensors positioning. The approaches covered for the analysis includes the grid based approach, physical position estimation approach and the stress analysis approach. Theory and concepts along with the examples of the algorithms proposed using these approaches were presented. The reviewed strategies each have their own benefits or costs.

Author [3] also explored the coverage concept under different parameters so that effective network deployment will be done. Author has identify the parameters involved in the coverage including the connectivity analysis and energy level comparison. Once the parameters will be collected, these properties will be combined under classification methods to obtain the coverage level analysis. This property includes point level coverage estimation, block level estimation and the barrier point identification approach. Author has explored all these methods under different algorithmic approaches. Author [4] defined a work on static estimation to the network deployment under location uncertainty. Author has analyze the network for random positioning of nodes as well as targets and perform the energy level estimation with different resource constraints. These constraints include the resource restriction so that network life will be improved. Author identified the vectors based on which reliable and energy effective coverage can be obtained over the network. Author[5] presented a study based work on coverage estimation under different design problems of sensor network. Author analyzed the core constraints for network generation so that the long term challenges will be covered. These constraints include the connectivity analysis, load analysis, energy effectiveness analysis and coverage range analysis. Author presented the work in the form of study as well as comparative analysis so that effective area coverage will be obtained over the network.

Various algorithms are proposed by the researches in order to optimize the problem. Disjoint and Non-Disjoint approaches are used to generate the cover sets which are used to cover specific targets. In Disjoint sets there is no intersection among the sets, the sensors are allowed to participate just in one spread set. This approach does not increase the network time viably. In Non-Disjoint approach the sensor may participate in more than one spread sets. This approach increases the network lifetime if proper scheduling algorithm is used. Author[6] defined work specific to resolve the problem of target coverage and provided the solution in terms of improved network life. Author has presented a weighted approach under coverage analysis on different constraints. These constraints generated the set of vectors based on which the effective network coverage can be obtained and the network life can be improved/ Author also presented the estimation under polynomial time analysis so that network life will be improved over the network. Author[7] maximize the network lifetime for target coverage problem by organizing the sensors into maximum disjoint set covers and these are activated successively. Author[8] focused on one of the major issues of target coverage problem of sensor network that is maximize the network lifetime, which can be solved by selecting minimum working nodes that will cover all the targets. The main objective is to determine the coverage ratio of all targets which have been significantly improved in comparison with the basic ant colony algorithm. The target coverage problem has been optimized by making it energy efficient using modified ant colony algorithm.

Author[9] planned associate degree energy-balance heuristic distributed formula supported energy utility of sensors. The target coverage issue with adjustable sensing ranges is reworked into the h-jump native target coverage issue with adjustable sensing ranges. Besides, he thought-about the key constraint of network life by introducing the definition of key target, define the vitality utility capability supported the quantitative relation of target coverage commitment to vitality utilization value and establish the reconciling adjustment mechanism of waiting time. The simulation shows that the planned formula will prolong greatly the network life and has lower machine quality and communication quality, sensible quantifiability and stability. Author [10] investigated life optimization for target coverage in wireless detector network with QoS necessities during this approach a column resembling possible answer is generated. Author[11] planned a heuristic greedy optimum coverage formula to maximize network life for target coverage. Foremost they analyzed the energy model for target coverage and conferred the definition of key target and therefore the coverage priority of key target. Then a technique for detector choice during which the detector with a lot of energy utility is previous chosen as active detector is meant. Then the formula is planned supported minimizing the energy consumption of key target and maximizing energy potency of detector node. The formula is extremely effective and sensible climbable. [12][13].

\section{CHALLENGES IN NETWORK COVERAGE}

There are units many factors that has got to be thought-about once developing an inspiration for coverage in a very sensing element networks. Several of those are dependent upon the actual application that's being self-addressed. The capabilities of the sensing element nodes that area unit getting used should even be thought-about. Most researchers specialize in one preparation model however there are a unit papers that decide to develop an additional general algorithmic rule which will be employed in many varieties of preparation.

\subsection{Deployment}

A device network preparation will typically be classified as either a dense preparation or a distributed preparation. A dense preparation contains a comparatively high variety of device nodes within the given field of interest whereas a 
distributed preparation would have fewer nodes. The dense preparation model is employed in things wherever it's necessary |vital| \{important $\}$ for each event to be detected or once it's important to possess multiple sensors cowl a district. distributed preparations could also be used once the value of the sensors create a dense deployment preventative or after you wish to attain most coverage mistreatment the clean minimum variety of sensors. In most of the work finding out coverage it's assumed that the device nodes square measure static, they keep within the same place once they're deployed. device network nodes square measure deployed in a district by either inserting them in planned locations or having the nodes indiscriminately set [3][4].

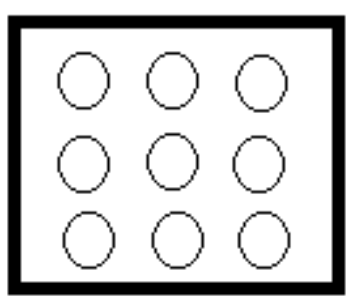

Figure 1.Deterministic deployment

In settled readying the position of every node and topology of the network is preset. Nodes are additional or deleted so as to regulate the density of the nodes and improve the coverage result of the network. Settled sensing element placement is applied to little to medium Senor Network during a friendly atmosphere. Figure 1 shows the instance of settled readying.

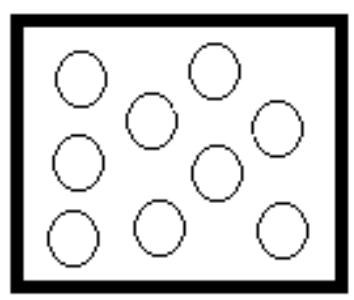

Figure 2.Random deployment

In random preparation the exact position and therefore the no. of device nodes can't be built or preset. Figure a pair of shows the instance of random preparation. during this device nodes square measure distributed inside the sector stochastically and severally it's sometimes for dangerous or abominable like battle field and foe military and disaster application or in hospitable areas wherever network size is massive. The sensors need to be at random deployed into the inclined space,

Wherever they acknowledge the targets, observe them and send the observation knowledge back to the sink via Multihop communications. Dropping sensors from a plane would be AN example of random placement.

\subsection{Node Types}

The collection of nodes that are hand-picked for a detector network are often either a uniform or heterogeneous cluster of nodes. a uniform cluster could be a cluster within which all the nodes have an equivalent capabilities (like same sensing vary, communication range). A heterogeneous cluster is that the one within which some nodes are additional powerful than the opposite nodes. Cluster of additional powerful nodes called cluster heads would gather information from the less powerful nodes many algorithms for best coverage mistreatment homogeneous nodes are given in [7]. Any algorithmic rule which can work for a heterogeneous network also will work with homogeneous network.

\subsection{Constraints}

There square measure varied kinds of constraints that square measure taken into thought for coverage in an exceedingly detector network. a number of the constraints square measure shortly printed below

\subsubsection{Energy constraint}

The most important factor to be considered in the development of a coverage scheme is that of energy constraints. Since sensor nodes are battery powered having limited energy capacity. Energy presents a big gainsays for network designers in hostile environment where it is impossible to access the sensors and recharge their batteries. When the energy of a sensor reaches a certain threshold level, the sensor will become defective and will not be able to function properly, which will affect the network performance up to a great extent. Therefore it becomes very important to conserve energy and prolong battery life[12].

\subsubsection{Connectivity constraint:}

Connectivity is an important issue in WSNs which concerns with delivering the sensed data from the source sensor to the destination (sink node) via radio transmissions. Connectivity refers to network connectivity and hence requires that all sensors in the network are connected to each other. For maintaining coverage with connectivity each active sensing node must be connected to the Base Station(sink) i.e. there must exists a route traversing through all active sensors and base station. Connectivity has inverse relationship with coverage. While coverage can be increase by not placing the sensors too close to each other the connectivity requires the sensors not to be placed too far apart. As sensors are low-cost devices with constrained resources, each sensor node has only limited communication range compared with the size of the monitored area. Multi-hop communications are necessary when a sensor cannot reach the sink node directly. Two sensors are called neighbors if they are within each other's communication range. The sensor nodes and the communication links between each pair of neighbors build the network topology, which is required to be connected by the connectivity requirement[1][2].

\subsubsection{QOS constraint:}

QoS is a wide and generalized terms that actually represents the network effectiveness under different parameters. These constraints includes the sensing quality analysis, network life analysis, communication rate analysis, sampling rate analysis, network reliability analysis, network lifetime analysis and failure rate analysis. These all constraints individually as well as collectively represents the network QoS.

\subsection{Centralized/Distributed Algorithms}

The Coverage algorithms projected area unit either centralized or seized and localized. In a very centralized coverage algorithmic program the observance is at the start calculated on the bottom station and it's then sent to the device hubs for execution. The algorithmic program is run on one or additional hubs in a very centralized location sometimes close to the info sink and knowledge from all hubs have to be compelled to be transferred to the central hub the advantage of this approach is that it obliges low process force from the device hubs, that sometimes have restricted process capabilities [10][11]. 
In distributed coverage algorithms, numerous device hubs perform the duty-bound calculations hand in glove then these hubs flow into the programing info to no matter remains of the sensors. the coverage algorithmic rule is dead supported the knowledge from just a few nodes(e.g., neighboring hubs at intervals a continuing range of trusts) in WSN and also the alternative is formed regionally because the alternative procedure is decentralized . Localized algorithmic rule suggests that several or the entire hub run the algorithmic rule singly on the knowledge every has gathered. These plans might oblige some process by the sensors concerned, but they scale higher to accommodate larger networks [10][13].

\section{CONCLUSION}

In this paper, the exploration to area coverage is defined. The paper has discussed, various issues associated with different kind of challenges and constraints associated with the coverage algorithms. The issues considered in this work includes deployment of nodes, node type and the associated constraints such as energy, connectivity etc. The paper also discussed the concept of centralized and decentralized network under consideration. The work is defined as a study based work based on which target coverage algorithm will be defined in future. The future work will analyze the network reliability under these vectors and provide the significant optimization to the network parameters.

\section{REFERENCES}

[1] GholamAli Yaghoubi," Connectivity Issue in Wireless Sensor Networks by Using Depth-First Search and Genetic Algorithm", 2010 International Conference on Computational Intelligence and Communication Systems 978-0-7695-4254-6/10 @ 2010 IEEE (pp 377-381)

[2] Nauman Aslam," Distributed Coverage and Connectivity in Three Dimensional Wireless Sensor Networks", "IWCMC'10, June 28- July 2, 2010, Caen, France. Copyright (C) 2010 ACM 978-1-4503-0062 9/10/06 (pp 1141-1145)

[3] Yinian Mao," Coordinated Sensor Deployment for Improving Secure Communications and Sensing Coverage", SASN'05, November 7, 2005, Alexandria, Virginia, USA. ACM 1595932275/05/0011 (pp 117128)

[4] Chi-Fu Huang," The Coverage Problem in a Wireless Sensor Network", WSNA'03, September 19, 2003, San Diego, California, USA. Copyright 2003 ACM 1-58113764-8/03/0009 (pp 115-121)

[5] Ashwinkumar Badanidiyuru," Approximating LowDimensional Coverage Problems", SCG'12, June 17-20, 2012, Chapel Hill, North Carolina, USA. ACM 978-14503-1299-8/12/06 (pp 161-170)
[6] Vikram P. Munishwar," Coverage Management for Mobile Targets in Visual Sensor Networks", MSWiM'12, October 21-25, 2012, Paphos, Cyprus. ACM 978-1-4503-1628-6/12/10 (pp 107-115)

[7] Vijay Chandrasekhar," Localization in Underwater Sensor Networks - Survey and Challenges", WUWNet'06, September 25, 2006, Los Angeles, California, USA. ACM 1-59593-484-7/06/0009 (pp 3340)

[8] Muzammil Hussain," Distributed Localization in Cluttered Underwater environments", WUWNet'10, Sept. 30 - Oct. 1, 2010, Woods Hole, Massachusetts, USA ACM 978-1-4503-0402-3

[9] Melike Erol," Localization with Dive'N'Rise (DNR) Beacons for Underwater Acoustic Sensor Networks", WUWNet'07, September 14, 2007, Montréal, Québec, Canada. ACM 978-1-59593-736-0/07/0009 (pp 97-100)

[10] M Bala Krishna," Computing Methodologies for Localization Techniques in Wireless Sensor Networks", International Conference and Workshop on Emerging Trends in Technology (ICWET 2011) - TCET, Mumbai, India ICWET'11, February 25-26, 2011, Mumbai, Maharashtra, India. ACM 978-1-4503-0449-8/11/02 (pp 1024-1028)

[11] Diba Mirza," Real-time Collaborative Tracking for Underwater Networked Systems", WUWNet'12, Nov. 5 - 6, 2012 Los Angeles, California, USA. ACM 978-14503-1773-3/12/11

[12] Sangbo Seo ," A New Energy Efficient Data Transmission Method for Underwater Wireless Sensor Networks",CSTST 2008, October 27-31, 2008, CergyPontoise, France. ACM 978-1-60558-046-3/08/0003 (pp 675-681)

[13] Liangjie He," Implementation and Emulation of Distributed Clustering Protocols for Wireless Sensor Networks", IWCMC'07, August 12-16, 2007, Honolulu, Hawaii, USA. ACM 978-1-59593-695-0/07/0008 (pp 266-271)

[14] Santosh Kumar," Barrier Coverage With Wireless Sensors", MobiCom'05, August28-September 2, 2005, Cologne, Germany. ACM 1-59593-020-5/05/0008 (pp 284-298)

\section{ACKNOWLEDGEMENT}

I would like to thank to my m.tech advisors, Assistant Professor Suman Bhatia, Department of Computer Science and Technology, ITMU, Gurgaon, India, for supporting me during my m.tech. Suman has been helpful in providing advice many times during my postgraduate career. She was and remains my best role model mentor, and teacher. 